\title{
A new Approach to Tricyclic Antidepressants Detection Based on Graphite Microsensors Fabricated by an Innovative Method
}

\author{
Beata Rozum ${ }^{1}$, Marek Dawgul', Jerzy Kruk ${ }^{1}$, Władysław Torbicz ${ }^{1}$, Dorota G. Pijanowska ${ }^{1}$ \\ ${ }^{1}$ Nalecz Institute of Biocybernetics and Biomedical Engineering, Polish Academy of Sciences, \\ 4 Trojdena St., 02-109 Warsaw, Poland \\ brozum@ibib.waw.pl
}

\begin{abstract}
In this study a novel low-cost method of amperometric sensors' fabrication based on thick-film technique on solid support and their analytical application are presented. Complete graphite/silver cell sensors were fabricated with the new technology using a desk-top XYZ-dispensing robot and printing pastes. The amperometric sensors were applied for the detection of three selected tricyclic antidepressant drugs (TCAs): imipramine (IMI), amitriptyline (AMI) and doxepin (DOX). The monitoring of TAC concentration in patients' body fluids is need, because these drugs can cause side or even toxic effect when overdosed. The possibility of simultaneous and individual amperometric determination of these compounds in media of different $\mathrm{pH}$ is demonstrated. The highest sensitivity was obtained for imipramine $(213 \mathrm{nA} / \mu \mathrm{M})$, while for doxepin and amitriptyline sensitivity was similar 32 and $34 \mathrm{nA} / \mu \mathrm{M}$, respectively. Moreover, electrochemical determination of imipramine was characterized by the lowest potential of peak current $(+0.77 \mathrm{~V})$.
\end{abstract}

Key words: sensors fabrication, amperometric sensors, tricyclic antidepressant drugs, imipramine, amitriptyline, doxepin.

\section{Introduction}

Tricyclic antidepressant compounds belong to the group of psychotropic drugs, which are widely applied for affective or 'mood' disorders treatment (depression, neurosis, bulimia nervosa, etc.). The mechanism of TCAs action is attributed to binding to 5 HT and noradrenaline re-uptake transporters in brain [1]. Despite their therapeutic action, TACs can cause serious side-effects including: chest pain, blurred vision and panic attacks. Toxic-effects can be induced especially when overdosed and/or drugs high-dose is combined with alcohol [2]. Therefore, the detection of TAC concentration in patients' body fluids became an important analytical task.

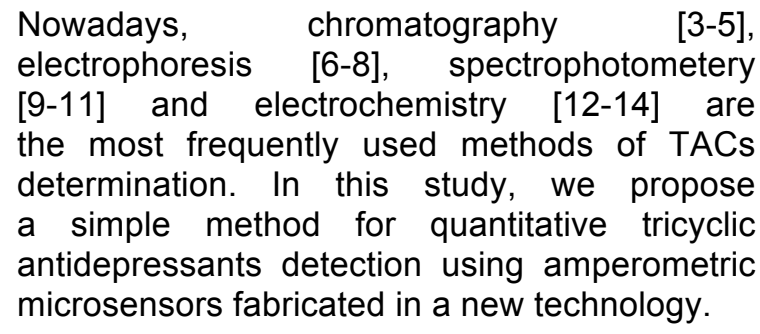

\section{The Fabrication of Electrochemical Sensors}

A variety of substances that might be determined using electrochemical sensors forces ongoing research concerning electrodes materials and methods of their deposition. In our laboratory a novel method based on a desk-top XYZ-dispensing robot (325 Ultra TT, EFD, USA) for deposition of different materials in a liquid form was developed. The robot allows printing lines down to $0.2 \mathrm{~mm}$ width and ca. $0.05 \mathrm{~mm}$ thick as well as complex geometric patterns for electrical contact pads, conductive paths, sensing areas and insulation layers.

In this experiment, we set our equipment for production of up to 96 sensors in one automatic dispensing cycle. The sensors structures were obtained by the deposition of 3 layers, consecutively: silver (5000 DuPont), graphite (L 951, ITME, Poland) and insulation (7165 DuPont) onto a polyester foil (Autostat CT7). Each layer was dried at temperature $120-130^{\circ} \mathrm{C}$ for $15 \mathrm{~min}$. A full compatibility of commercially available materials designed for screen-printing with this equipment was demonstrated. 
Main advantage of a novel deposition method over screen-printing is reduction of deposited material lost. The printing pastes are transferred from a disposable applicator thru a dispensing tip onto substrate accordingly to prior designed pattern. The excessive material can be returned from the applicator to the storage container and laborious and solvent-consuming cleaning of the equipment (screens, drivers) becomes unnecessary. The sensor design can be easily modified by means of software without generation of additional costs of photographic masks, screens etc. This technique can be used for deposition of various materials such as: pastes, inks, emulsions, epoxy and silicone resins and organic solutions on different types of substrates e.g. polymeric foils, glass, ceramics and many others. Additional advantages of desk-top XYZ-dispensing robot utilization are flexibility in design and pattering applicable for both rapid prototyping of test samples and mass production. The same equipment configuration can be used independently of production scale.

\section{Reagents and Methods}

Tricyclic antidepressant drugs: imipramine, amitriptyline and doxepin as hydrochlorides were obtained from Sigma. Drugs stock solutions $(0.1 \mathrm{M})$ were prepared in water and stored at $+5^{\circ} \mathrm{C}$ in the dark. Calibration standards were prepared just before experiments by the dilution of stock with running buffer to desired concentrations daily prior to use. All other reagents were of analytical grade. All solutions used throughout the experiments were prepared using ultrapure water (resistivity of $18 \mathrm{M} \Omega$ ) made with Milipore system. Before use the solutions were deoxidized with gaseous nitrogen.

Measurements were carried out using 3-electrode sensors with graphite layers as working and counter electrodes and silver chloride layer as a reference electrode. The silver chloride electrode was formed by electrochemical chlorination of silver path. Voltamperometric measurements were performed using PalmSens potentiostat (Palm Instruments BV, The Netherlands) employing: cyclic voltammetry (CV) and linear sweep voltammetry (LSV). All measurements were carried out at room temperature.

\section{Results}

As mentioned above, the fabricated amperometric sensors were employed for electrochemical quantification of three selected
TCAs, namely: imipramine (IMI), amitriptyline (AMI) and doxepin (DOX). The difference between those three substances consists in heterocyclic rings.

The first attempts were made to choose the best chemical and electrochemical conditions for performing quantification of individual TCAs. The detection of each TAC was investigated in wide concentration range using cyclic voltammetry. $50 \mathrm{mM}$ phosphate buffer $\mathrm{pH} 7$ $(0.1 \mathrm{M} \mathrm{KCl})$ was chosen as an optimal solution for electrochemical detection for all determined TCAs. Cyclic voltammograms were recorded in a potential range from 0 to $1.2 \mathrm{~V}$ with the scan rate of $100 \mathrm{mV} / \mathrm{s}$. Exemplary CV curves for different IMI concentrations and corresponding calibration curve are presented in Figure 1 . It was found that the potential of peaks current for IMI, AMI and DOX were $+0.77 \mathrm{~V},+0.97 \mathrm{~V}$ and $+0.98 \mathrm{~V}$, respectively. Analytical parameters which specify the determination of selected TACs are compiled in table 1. Parameters were defined for drugs over the concentration range from 0.1 to $10 \mathrm{mM}$, which include plasma TACs level $[15,16]$. The highest sensitivity of the amperometric graphite sensors was obtained for imipramine - $213.0 \mathrm{nA} / \mu \mathrm{M}$, while for doxepin and amitriptyline was comparable, namely $32.0 \mathrm{nA} / \mu \mathrm{M}$ and $34.2 \mathrm{nA} / \mu \mathrm{M}$, respectively.

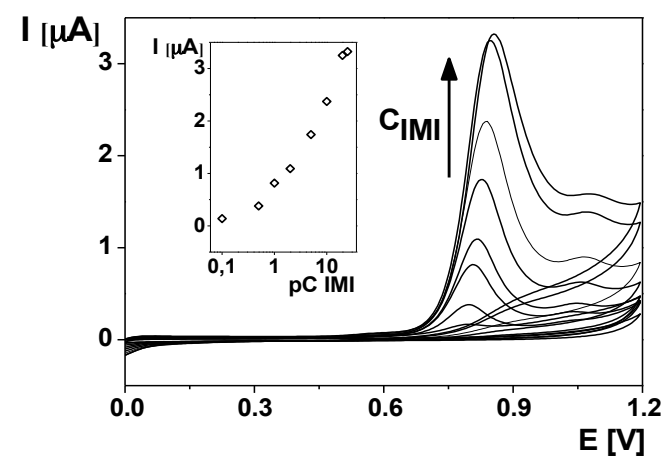

Fig. 1. Typical cyclic voltamperograms for IMI and corresponding calibration graph (inset). Potential range: 0 - $1.2 \mathrm{~V}$, scan rate: $100 \mathrm{mV} / \mathrm{s}$, incubation time: 2 min.

The proposed approach under optimal conditions was adapted for the simultaneous detection of IMI, AMI and DOX in mixture. Linear sweep voltammograms for mixtures of drugs in different concentration recorded in $50 \mathrm{mM}$ phosphate buffer $\mathrm{pH} 7,0.1 \mathrm{M} \mathrm{KCl}$ are shown in Figure 3. As can be seen the potential of imipramine oxidation stays unchanged, whereas for AMI and DOX one peak consisting of two merged peaks current is recorded. It is worth noticing, that the peak current for IMI 
is amplified in mixture comparing to individual drug determination (compare Figures 2 and 3 ).

Tab. 1: Analytical characteristic of proposed detection method for three selected TACs in concentration ranged from 0.1 to $10 \mu \mathrm{M}$. Optimal conditions: $50 \mathrm{mM}$ phosphate buffer $\mathrm{pH} 7,0.1 \mathrm{M} \mathrm{KCl}$. Cyclic voltammetry, potential range: $0.0 \mathrm{~V}-1.2 \mathrm{~V}$, scan rate $100 \mathrm{mV} / \mathrm{s}$, Estep $5 \mathrm{mV}$. Drug incubation time: 2 min.

\begin{tabular}{|r|c|c|c|}
\hline \multirow{2}{*}{$\begin{array}{c}\text { Analytical } \\
\text { parameter }\end{array}$} & \multicolumn{3}{|c|}{ Tricyclic Antidepressant } \\
Drug \\
\cline { 2 - 4 } & IMI & AMI & DOX \\
\hline $\begin{array}{r}\text { Sensitivity } \\
{[\mathrm{nA} / \mu \mathrm{M}]}\end{array}$ & 213.0 & 34.2 & 32.0 \\
\hline $\begin{array}{r}\text { Oxidation } \\
\text { Potential [mV] }\end{array}$ & 770 & 970 & 980 \\
\hline $\mathrm{R}^{2}$ & 0.9161 & 0.9598 & 0.9853 \\
\hline
\end{tabular}

Next, a linear sweep voltammetry for each drug was performed (Figure 2).

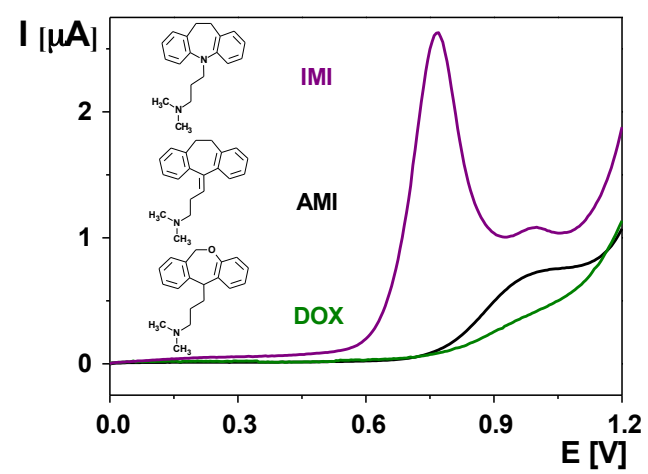

Fig. 2. Typical linear sweep voltamperograms for selected TACs (depicted in the graph). Linear sweep voltammetric response recorded for drugs of $10 \mu \mathrm{M}$ concentration in $50 \mathrm{mM}$ phosphate buffer $\mathrm{pH} \mathrm{7,} 0.1$ $\mathrm{M} \mathrm{KCl}$.

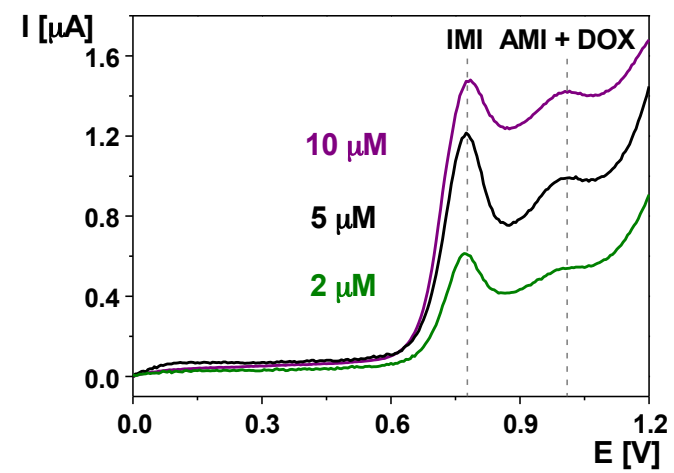

Fig. 3. Simultaneous detection of IMI, AMI, DOX of different concentrations $(2,5$ and $10 \mu \mathrm{M}$ of each drug) in mixture.
Finally, it was proved that selectivity for IMI in TCAs mixture can be improved by use of a buffer solution of appropriate $\mathrm{pH}$. For comparison, in figure 4, linear sweep voltammograms for the mixtures of the three drugs in solutions at $\mathrm{pH} 2$ and 7 are presented. We found that electrochemical oxidation takes place also in buffer solutions of $\mathrm{pH} 2$ for imipramine only. However, the determination of imipramine in solutions of $\mathrm{pH} 2$ is characterized by lower sensitivity.

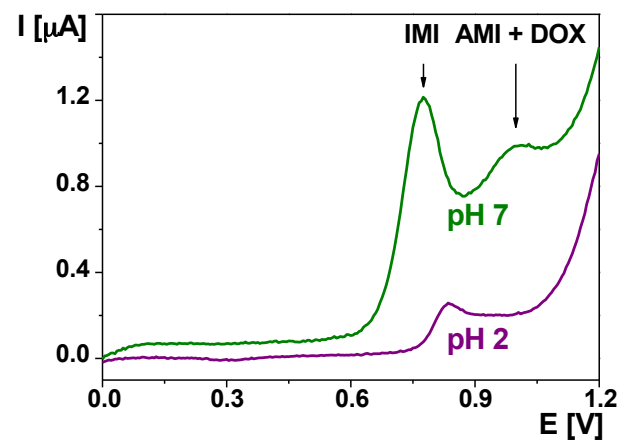

Fig. 4. Simultaneous detection of IMI, AMI, DOX of $5 \mu \mathrm{M}$ concentration in mixture in buffers of different $\mathrm{pH}$ (depicted in the graph).

\section{Conclusions}

In this study, we presented an innovative method for the fabrication of thick-film sensors on solid support using a desk-top XYZ-dispensing robot. The advantages of proposed method were discussed.

The complete electrochemical cells consisting of graphite working and counter electrodes and silver/silver chloride reference electrode were shown to be suitable for the detection of tricyclic antidepressant drugs. The highest sensitivity $(213 \mathrm{nA} / \mu \mathrm{M})$ and the lowest potential of peak current $(+0.77 \mathrm{~V})$ were obtained for imipramine. In addition, the possibility of imipramine, amitriptyline and doxepin quantification independently as well as in the mixtures was presented.

\section{Acknowledgements}

This research was financed by the EU (European Regional Development Found) within the POIG Program: MNS-DIAG "Microand Nano- Systems for Chemistry and Biomedical Diagnostics" (POIG.01.03.0100-014/08-02). 


\section{References}

[1] M. Hamon, S. Bourgoin, Pharmacological Profile of Antidepressants: a Likely Basis for Their Efficacy and Side Effects?, European Neuropsychopharmacology 16, S625-S632 (2006); doi: 10.1016/S0924-977X(06)70008-6

[2] E. Tanaka, T. Nakamura, M. Terada, K. Honda, An in vitro Study on the Interaction Between Ethanol and Imipramine at High Concentrations Using Human Liver Microsomes, Forensic Toxicology 25, $96 \quad-\quad 99 \quad$ (2007); doi: $10.1007 / \mathrm{s} 11419-007-0036-4$

[3] M.A. Martínez, C. Sánchez de la Torre, E. Almarza, Simultaneous Determination of Viloxazine, Venlafaxine ,Imipramine, Desipramine, Sertraline, and Amoxapine in Whole Blood: Comparison of Two Extraction/Cleanup Procedures for Capillary Gas Chromatography with Nitrogen-Phosphorus Detection, Journal of Analytical Toxicology 26, 296-308 (2002); doi: 10.1093/jat/26.5.296

[4] D. Badenhorst, F.C.W. Sutherland, A.D. de Jager, T. Scanes, H.K.L. Hundt, K.J. Swart, A.F. Hundt, Determination of Doxepin and Desmethyldoxepin in Human Plasma Using Liquid Chromatography-Tandem Mass Spectrometry, Journal of Chromatography B 742, 91-98 (2000); doi: 10.1016/S03784347(00)00136-5

[5] P.P. Rop, A. Viala, A. Durand, T. Conquy, Determination of Citalopram, Amitryptyline and Clomipramine in Plasma by Reversed-Phase High-Performance Liquid Chromatography, Journal of Chromatography - Biomedical Applications $\quad 338, \quad 171-178 \quad$ (1985); doi: 10.1016/0378-4347(85)80081-5

[6] C. Yu, H. Du, T. You, Determination of imipramine and trimipramine by capillary electrophoresis with electrochemiluminescence detection, Talanta 83, 1376-1380 (2011), doi: 10.1016/j.talanta.2010.11.011

[7] C.-C. Chen, S.-M. Wu, Y.-H. Huang, W.-K. Ko, H.-S. Kou, H.-L. Wu, On-Line Field-Amplified Sample Stacking in Capillary Electrophoresis for Analysis of Amitriptyline and its Metabolite Nortriptyline in Plasma, Analytica Chimica Acta 517, 103-110 (2004); doi: 10.1016/j.aca.2004.05.002

[8] C.-S. Liu, X.-F. Li, D. Pinto, E. B. Hansen Jr., Carl E. Cerniglia, N. J. Dovichi, On-Line Nonaqueous Capillary Electrophoresis and Electrospray Mass
Spectrometry of Tricyclic Antidepressants and Metabolic Profiling of Amitriptyline by Cunninghamella Elegans, Electrophoresis 19, 3183-3189

(1998); doi: $10.1002 /$ elps.1150191822

[9] W. Misiuk, Spectrophotometry Assay of Imipramine and Desipramine Using Ammonium Metavanadate and its Application to Pharmaceutical Preparations, Journal of Pharmaceutical and Biomedical Analysis 22, 189-196 (2000); doi: 10.1016/S07317085(99)00287-3

[10] W. Misiuk, Extractive spectrophotometric methods for the determination of doxepin hydrochloride in pharmaceutical preparations using titanium (IV) and iron (III) thiocyanate complexes, II Farmaco 60, 61-69 (2005); doi: 10.1016/j.farmac.2004.09.004

[11] E.M. Elnemma, F.M. El Zawawy, S.S.M. Hassan, Determination of Amitriptyline, Imipramine and Orphenadrine in Antidepressant Drugs by Potentiometry, Spectrophotometry and Atomic Absorption Spectrometry, Mikrochimica Acta 110, 79-88 (1993); doi: 10.1007/BF01243988

[12] E.S. Smotkin, G.B. Martin, G.A. Rechnitz, Electrochemical Determination of Tricyclic Antidepressants, Analytical Letters 24, 797-808 (1991); doi: 10.1080/00032719108052943

[13] X.-L. Xu, F. Huang, G.-L. Zhou, S. Zhang, J.-L. Kong, A Novel Electrochemical Sensor for Probing Doxepin Created on a Glassy Carbon Electrode Modified with Poly(4-Amino- benzoic Acid)/Multi-Walled Carbon Nanotubes Composite Film, Sensors 10, 8398-8410 (2010) doi:10.3390/s100908398

[14] K. Madej, P. Kościelniak, Review of Analytical Methods for Identification and Determination of PHEs and Tricyclic Antidepressants, Critical Reviews in Analytical Chemistry 38, 51-67 (2008); doi: 10.1080/10408340701804343

[15] G. Silverman, R. A. Braithwaite, Benzodiazepines and Tricyclic Antidepressant Plasma Levels, British Medical Journal 3, 18-20 (1973); doi: $10.1136 / \mathrm{bmj} .3 .5870 .18$

[16] S. Vandel, G. Bertschy, B. Bonin, S. Nezelof, T.H. Francois, B. Vandel, D. Sechter, P. Bizouard, Tricyclic antidepressant plasma levels after fluoxetine addition, Neuropsychobiology 25, 202-207 (1992); doi: 10.1159/000118833 